\title{
Décadrages Décadrages
}

cınéma, à travers champs Cinéma, à travers champs

$16-17$ | 2010

Les abîmes de l'adaptation

\section{La vie «fantastique » des frères Grimm : une biographie fictive par Terry Gilliam}

\section{Cyrille François}

\section{OpenEdition}

\section{Journals}

Édition électronique

URL : http://journals.openedition.org/decadrages/240

DOI : $10.4000 /$ decadrages. 240

ISSN : 2297-5977

Éditeur

Association Décadrages

Édition imprimée

Date de publication : 1 février 2010

Pagination : 95-103

ISBN : 978-2-9700668-1-1

ISSN : 2235-7823

Référence électronique

Cyrille François, "La vie « fantastique » des frères Grimm : une biographie fictive par Terry Gilliam », Décadrages [En ligne], 16-17| 2010, mis en ligne le 10 février 2011, consulté le 21 avril 2019. URL :

http://journals.openedition.org/decadrages/240 ; DOI : 10.4000/decadrages.240 


\section{La vie "fantastique " des frères Grimm : une biographie fictive par Terry Gilliam}

par Cyrille François

Lassociation du nom de Terry Gilliam à un projet de film sur les frères Grimm semblait une évidence tant les mondes merveilleux créés par le réalisateur de Time Bandits (Bandits, bandits, 1981), Brazil (1985) ou encore The Adventures of Baron Munchausen (Les aventures $d u$ baron de Münchausen, 1988) exploitent un imaginaire proche de ceux des contes de fées ${ }^{1}$. On pouvait toutefois s'attendre à ce que ce projet soit plus qu'un biopic sur Jacob et Wilhelm Grimm², auteurs des Kinder- und Hausmärchen (Contes de l'enfance et du foyer, première édition en 1812). Tel est en effet le cas puisque, si le titre "The Brothers Grimm» (Les frères Grimm, 2005) et les premières scènes du film pourraient laisser penser que ce dernier raconte la vie des auteurs allemands, le scénario d'Ehren Kruger ${ }^{3}$ s'écarte de la biographie, dépeignant les Grimm sous les traits d'escrocs utilisant leurs connaissances des croyances populaires pour abuser leurs contemporains. La référence aux deux frères est donc surtout prétexte à une mise en images du monde merveilleux associé à leur nom, à une représentation basée sur des phénomènes de réception plus que sur une lecture fidèle de leurs textes. Par ailleurs, elle occasionne une transposition du genre du conte dans le cadre d'une intrigue fantastique. Ni biopic, ni adaptation d'un texte des Grimm, The Brothers Grimm offre toutefois une mise en scène de l'écriture et de l'objet livre qui convoque d'une certaine façon le récit de la genèse des Contes de l'enfance et du foyer.

\section{Des textes à leurs représentations populaires}

The Brothers Grimm s'inspire très librement de plusieurs contes des Grimm qui sont tissés tout au long de l'intrigue filmique 4 . Plus que l'adaptation de textes, Gilliam propose une succession de références liées à l'imaginaire véhiculé par les contes. Bien qu'il assure avoir lu les Contes de l'enfance et du foyer et qu'il démontre une bonne connaissance de ceux-ci5, le réalisateur préfère se baser sur ce qui a marqué
1 C'est en partie cela qui a poussé Terry Gilliam à accepter ce qui est avant tout un film de commande dont il n'aimait pas le scénario - il le répète dans toutes les interviews et même dans le commentaire audio du dvd du film (Concorde Video, "Cine collection", 2006, édition 2 disques) - et pour lequel les producteurs Harvey et Bob Weinstein ne lui ont pas accordé le final cut. Ce film à grand budget était un moyen de rassurer les studios et de gagner de l'argent après une série de quatre films avortés. Pour Gilliam, ce devait être là aussi un moyen de terminer The Man Who Killed Don Quixote. Bob McCabe retrace les nombreux problèmes survenus au cours du tournage et les concessions faites par le réalisateur qui font dire à certains membres de l'équipe que The Brothers Grimm n'est pas vraiment un film de Terry Gilliam (Bob McCabe, Dreams and Nightmares: Terry Gilliam, "The Brothers Grimm" and Other Cautionary Tales of Hollywood, London, Harper Collins Entertainment, 2005).

2 A l'instar de Philippe Génot, qui a consacré à Terry Gilliam un article avant la sortie du film: Philippe Génot, "Allons cultiver notre Graal: contes et légendes chez Terry Gilliam ", in Carole Aurouet (éd.), CinémAction, n 116 ("Contes et légendes à l'écran "), Corlet, 2005, pp. 129-138.

3 Lancé avec le scénario d'Arlington Road (1999), Ehren Kruger s'est illustré dans le genre fantastique avec plusieurs succès: The Ring ( $L e$ cercle, 2002), The Ring Two (Le cercle 2, 2005), The Skeleton Key (La porte des secrets, 2005). II a aussi signé les scénarios de Scream 3 (2000) et des deux derniers volets de la trilogie Transformers (2009 et 2011).

4 Le commentaire audio du dvd et les notes de production filent les métaphores sur les couches et le tissage: "Tout au long de l'action ininterrompue du film, Gilliam tisse de manière ludique des fils issus des plus populaires des contes des Grimm." (je traduis; les notes de productions sont disponibles en anglais à cette adresse: http://thecia.com.au/reviews/ b/brothers-grimm.shtml).

5 Le making-of disponible sur le dvd du film montre par exemple que Gilliam est conscient du travail de réécriture des Grimm entre la première édition de leur recueil en 1812 et la dernière de leur vivant, en 1857. 


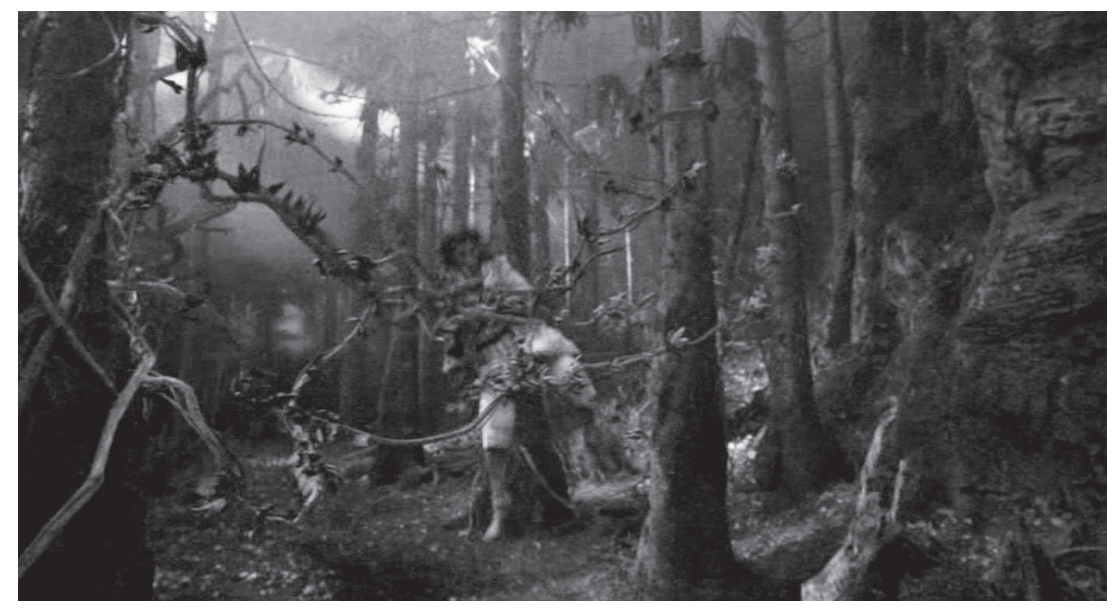

1

6 Texte tiré des notes de production. Un peu plus loin, les notes précisent (ce n'est cette fois pas une citation de Gilliam): "Aujourd'hui, ces histoires sont une part indélébile de la culture populaire".

7 Arthur Rackham (1869-1939), artiste britannique, a marqué des générations avec ses illustrations de contes de Grimm et d'autres classiques de Carroll, Swift ou encore Andersen.

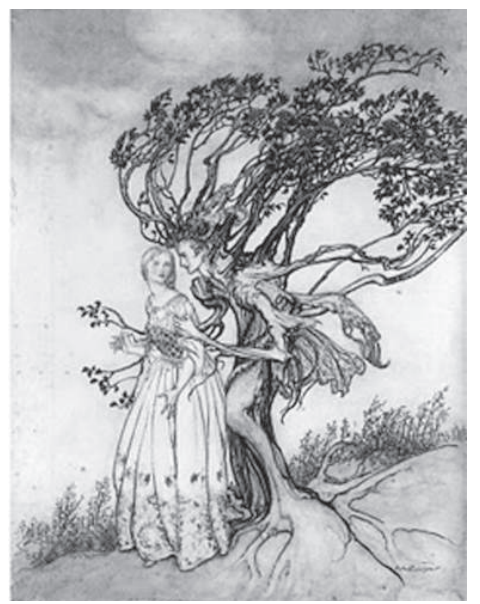

3 les esprits et sur ce que les contes sont susceptibles d'évoquer chez le spectateur:

«Bien qu'il y ait en fait plusieurs centaines de contes des Grimm, nous voulions nous limiter à ceux qui trouvent un écho dans l'imagination des gens. » 6

On apprend ainsi dans les commentaires audio de l'édition dvd Concorde Video que Gilliam s'est inspiré d'illustrations célèbres des contes, comme celle qu'Arthur Rackham a faite pour le conte «Die Alte im Wald» («La vieille dans la forêt») représentant des arbres animés 7 . Les arbres de la forêt enchantée de The Brothers Grimm, qui attaquent les héros et leur guide Angelika (fig. 1), ne sont pas non plus sans rappeler l'esthétique de ceux du Snow White and the Seven Dwarfs (Blanche-Neige et les sept nains, 1937) de Walt Disney (fig. 2), qui, eux aussi, sont des êtres inquiétants bien différents de l'arbre prince embrassant l'hérö̈ne

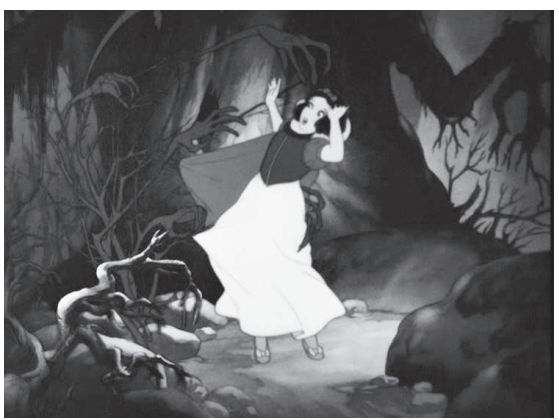

2 
entre ses branches dans le texte des Grimm (fig. 3) 8. Avec Cinderella (Cendrillon, 1950) et Sleeping Beauty (La belle au bois dormant, 1959), Snow White est l'un des phénomènes qui a transformé de manière radicale la réception des contes - nombreux sont les enfants qui ne connaissent que cette version via le film ou son adaptation en livre. De même, la scène de The Brothers Grimm faisant figurer une vieille femme avec une pomme rouge à la main est une référence à Blanche-Neige que le spectateur reconnaît immanquablement (fig. 4-5) 9 .

Les textes des Grimm sont en fait très mal connus dans le monde anglo-saxon (la situation n'est guère plus réjouissante en France). Dans un article de National Geographic, Thomas O'Neill indique que "la plupart d'entre nous ne les connaissent que dans leurs versions édulcorées »10. Peu savent en effet que ce n'est pas en recevant un baiser de la princesse que la grenouille de «Der Froschkönig oder der eiserne Heinrich » ( "Le roi-grenouille ou Henri-de-fer») se transforme en prince, mais en se faisant projeter contre un mur par l'héroïne. Dans «Schneewittchen» («Blanche-Neige») et «Dornröschen» («Rose d'épine», texte proche de «La belle au bois dormant» de Perrault), ce n'est pas le «baiser d'amour vrai» du "Prince Charmant» $\mathbf{1 1}$ qui réveille les héroïnes, mais, respectivement, le choc du cercueil heurtant le sol qui fait ressortir le bout de pomme empoisonné et les cent ans du mauvais sort qui se sont écoulés.

Les références aux contes des Grimm $\mathbf{1 2}$ dans le film de Gilliam sont ainsi souvent imprécises et mêlées à des éléments issus d'autres contes populaires dans le monde anglo-saxon («The Gingerbread Man»/«Le petit Bonhomme de pain d'épices»; "Jack and the Beanstalk»/«Jack et le haricot magique»; "Pinocchio»), voire avec des mythes et des légendes (les corbeaux d'Odin; la légende de la comtesse Elizabeth Bathory qui se baignait dans le sang de jeunes filles pour conserver sa jeunesse). Si les contes des Grimm sont finalement mal connus et si le réalisateur préfère
8 Arthur Rackham, Rackham's Fairy Tale Illustrations in Full Color, New York, Dover Publications, 2002, p. 4.

9 On pourrait encore relever l'expression "true love's kiss" ("baiser d'amour vrai"), titre d'une chanson de Sleeping Beauty utilisé à la fin de The Brothers Grimm; de même, les chaussures de verre évoquent une scène de Cinderella qui ne renvoie pas à un conte des Grimm, mais au "Cendrillon" de Perrault.

10 Thomas O'Neill, "Guardians of the Fairy Tale: The Brothers Grimm", National Geographic, décembre 1999, pp. 102-129 (je traduis). Disponible en ligne: www.nationalgeographic.com/ grimm/article.html (Accès le 11 avril 2010).

11 L'expression "Prince Charming" a d'ailleurs été popularisée par le Cinderella de Disney en 1950 et n'était pas utilisée à la manière d'un nom propre auparavant.

12 Voici les plus connus des contes auquel le film fait référence: "Rotkäppchen" ("Le petit chaperon rouge"), "Rumpelstilzchen", "Der Froschkönig oder der eiserne Heinrich ", "Hänsel und Gretel", "Die Alte im Wald", "Rapunzel" ("Raiponce"), "Schneewittchen", "Aschenputtel" (littéralement "Qui se roule dans les cendres", texte proche du "Cendrillon" de Perrault), "Dornröschen".

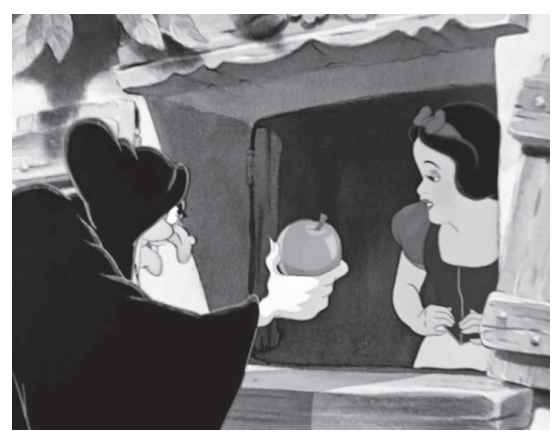

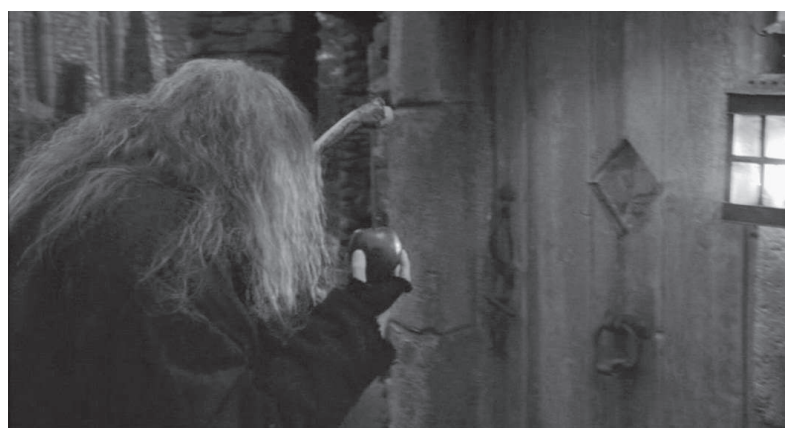

4 
13 Bob McCabe, op. cit., p. 25 (je traduis).

14 Jacob et Wilhelm Grimm, The Complete Fairy Tales of the Brothers Grimm [trad. par Jack Zipes], New York, Bantam Books, 2003, 3e édition, p. xxiii (je traduis).

15 Quand les notes de production parlent par exemple de "deux frères fringants mais diamétralement opposés", elles font référence aux auteurs tout en leur attribuant les caractères des personnages du film.

16 Le biographique semble d'ailleurs revendiqué, car le début se déroule en 1796, année de la mort du père des Grimm, qui entraîna des problèmes financiers (toutefois moins importants que ce que laisse penser le film).

17 Les notes de production insistent sur l'aspect folkloristique du travail des Grimm: "Les frères ont parcouru le pays, recueillant et propageant des contes", "[...] commencèrent à recueillir des contes populaires et des contes de fées - anciens contes transmis oralement de mères à leurs enfants", "[...] contes qu'ils ont recueillis de paysans allemands". Or, c'est précisément ce que les recherches remettent en question, comme le résume Zipes: "Contrairement aux croyances populaires, les Grimm n'ont pas recueilli leurs contes en rendant visite à des paysans à la campagne et en transcrivant les contes qu'ils avaient entendus " (Jacob et Wilhelm Grimm, op. cit., p. xxix). Depuis les années 1980, Heinz Rölleke a publié de nombreux travaux en allemand sur les Grimm, donnant une vision du travail accompli par les deux frères qui est plus fidèle à la réalité (pour un aperçu, voir Heinz Rölleke, Die Märchen der Brüder Grimm : eine Einführung, Stuttgart, Reclam, 2004).

18 Bob McCabe, op. cit., p. 16 (je traduis).

19 Sur le plan des faits, on pourrait objecter que c'est Jacob, et non Wilhelm, qui est le plus âgé des deux frères, ou encore que Karlstadt est plus proche de Frankfurt que Kassel, contrairement à ce qui est indiqué dans le film. On ne s'étonnera pas du fait que les crédits ne présentent pas de conseiller-expert des Grimm. faire appel aux souvenirs des lecteurs en s'adaptant aux connaissances du public américain, la situation est encore plus complexe en ce qui concerne les frères eux-mêmes. Nicola Pecorini, le directeur de la photographie, confie dans son journal ne pas avoir trouvé "grand chose sur les Grimm en tant qu'hommes» dans une grande librairie de Londres ${ }^{\mathbf{1 3}}$. Dans son introduction à l'édition complète des contes des Grimm en anglais, Jack Zipes confirme ces propos: "Curieusement, la plupart des critiques et des introductions des traductions anglaises des contes des Grimm disent peu de choses des frères eux-mêmes ou de leurs méthodes pour recueillir les contes $\gg \mathbf{1 4}$.

On se retrouve donc dans cette situation paradoxale où le nom des Grimm est très connu de par le monde sans que l'on sache qui ces derniers étaient réellement. Le film joue sur cette inconnue en mélangeant faits biographiques et fiction, avec parfois des risques de confusion $\mathbf{1 5}$. Le titre et le début du film laissent penser que le spectateur apprendra comment Jacob et Wilhelm Grimm sont passés d'un foyer pauvre au statut d'auteurs de l'un des livres les plus lus au monde16. De plus, lorsque les frères sont présentés comme des hommes parcourant l'Allemagne pour recueillir des histoires de la bouche du peuple, le film fait appel au savoir commun sur les Grimm. C'est là aussi une conception peu fidèle à la réalité, car les auteurs ont en fait très peu voyagé pour réaliser leur projet, et ont par ailleurs utilisé de nombreuses sources écrites $\mathbf{1 7}$. En outre, leurs informateurs ne provenaient, pour la plupart, pas du monde paysan, mais de la bourgeoisie et de l'aristocratie.

Le choix d'éviter la biographie n'est ainsi pas très étonnant, compte tenu du peu d'informations disponibles en anglais, mais surtout du fait que la vie des frères Grimm n'était pas aussi trépidante que ce à quoi l'on pourrait s'attendre dans un film d'action à grand budget. Il $\mathrm{y}$ a aussi, selon Bob McCabe, un phénomène de mode qui explique ce mélange de biographie et de fiction: «L'opportunité s'est présentée par un scénario d'Ehren Kruger qui ‘ré-imaginait〉 (un terme très en vogue à Hollywood à l'époque) les frères Grimm en un duo d'arnaqueurs du XVIII ${ }^{e}$ (sic) siècle»18. Il n'y a donc pas de volonté de raconter leur vie dans un souci de réalisme, car seule importe la possibilité de pénétrer dans le monde qu'ils ont créé19. Le mot d'ordre des notes de production est de présenter des personnages "fictionnalisés». On apprend ainsi que Terry Gilliam a développé les caractères des deux frères de manière antagoniste (Will le pragmatique et Jake le rêveur, avec leurs noms anglicisés), en réécrivant le scénario avec son ami et coscénariste Tony Grisoni pendant le tournage même. Le réalisateur avoue ainsi dans les notes de production: "Nous avons en fait créé un conte sur eux.». 


\section{Contes de fées et film fantastique}

Malgré cette affirmation de Gilliam, le film ne relève génériquement pas du conte de fées. Il se base certes sur plusieurs fragments de contes dont il reprend des motifs et renvoie à un imaginaire associé au genre, mais son intrigue et son rapport au surnaturel exploitent des codes du film fantastique 20. Généralement défini comme l'intrusion du surnaturel dans un récit réaliste, le fantastique se caractérise par l'expérience d'événements que les personnages ne peuvent expliquer selon les lois de la nature. Tzvetan Todorov insiste sur la notion d'hésitation, montrant que dans de tels récits, le lecteur, et parfois (mais pas toujours) les personnages, hésitent entre l'acceptation du surnaturel en tant que tel et la tentative d'explication rationnelle. Les contes de fées font quant à eux partie de la catégorie du "merveilleux pur» selon sa théorie: "les éléments surnaturels ne provoquent aucune réaction particulière ni chez les personnages, ni chez le lecteur implicite» $\mathbf{2 1}$. Ainsi, les héros des contes des Grimm ne s'étonnent pas de rencontrer des loups qui parlent et des personnages aux pouvoirs magiques, car le surnaturel est accepté comme allant de soi dans un monde qui n'est pas régi par les lois de la nature.

Dans The Brothers Grimm, des événements surnaturels pénètrent un monde réaliste et provoquent une hésitation chez les personnages et les spectateurs. L'univers merveilleux des contes est dans un premier temps remis en question: "C'est juste une histoire pour effrayer les enfants", indique un chasseur, séparant ainsi le merveilleux et la réalité. Les positions des personnages reflètent cette dichotomie, à l'instar de Jake, qui pense vivre dans un monde merveilleux où les haricots magiques existent et explique ce qui leur arrive selon cette croyance, et Will, qui est certain que tout est explicable rationnellement et que la forêt enchantée est le fait d'escrocs tirant parti des croyances populaires. Ces positions évoluent ensuite, laissant place au doute.

Tout le film est porté par cette tension entre réalité et merveilleux, comme le résume Nicola Pecorini lorsqu'il rend compte des consignes de Gilliam : "Nous devons être réalistes et nous assurer que le spectateur ne sache pas où se trouve la frontière entre réalité et merveilleux, de sorte qu'il puisse partager l'expérience des frères „22. L'intérêt du film réside ici dans le fait que la distinction entre réalité et merveilleux rejoint celle existant entre le réel et la fiction, puisque les éléments surnaturels qui surgissent dans le film sont issus des contes consignés par Jake et Will, ces derniers correspondant, par mise en abyme, aux textes qui furent effectivement publiés par Jacob et Wilhelm Grimm. Les protagonistes sont conscients de vivre l'une de ces histoires et parviennent à sauver le village par leurs connaissances des contes: "L'histoire, elle nous arrive
20 Gilliam indique d'ailleurs que le scénario original de Kruger était "un manuel de tous vos moments préférés de tous vos films fantastiques préférés" (Bob McCabe, op. cit., p. 22), avec des scènes lui rappelant The Mummy ( $L a$ momie, Stephen Sommers, 1999; voir Michael Tapper, "Beyond the banal surface of reality", Film International, février 2006, 4: 1, p. 63).

21 Tzvetan Todorov, Introduction à la littérature fantastique, Paris, Seuil, 1970, p. 59.

22 Bob McCabe, op. cit., p. 35. Les notes de production parlent de "bataille épique entre le merveilleux et la réalité " et jouent sur l'hésitation du spectateur: "[Les contes] ne sont pas réels. Ou le sont-ils?"; "les fantaisies les plus sauvages et les plus fabuleuses sont devenues... réalité". 


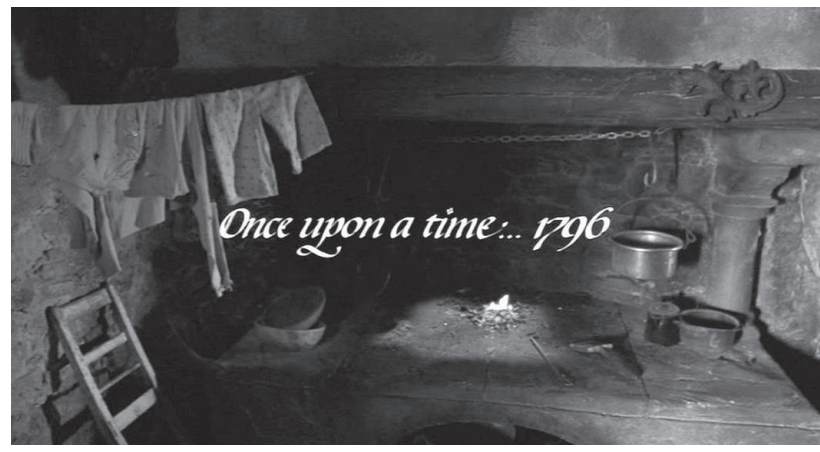

6

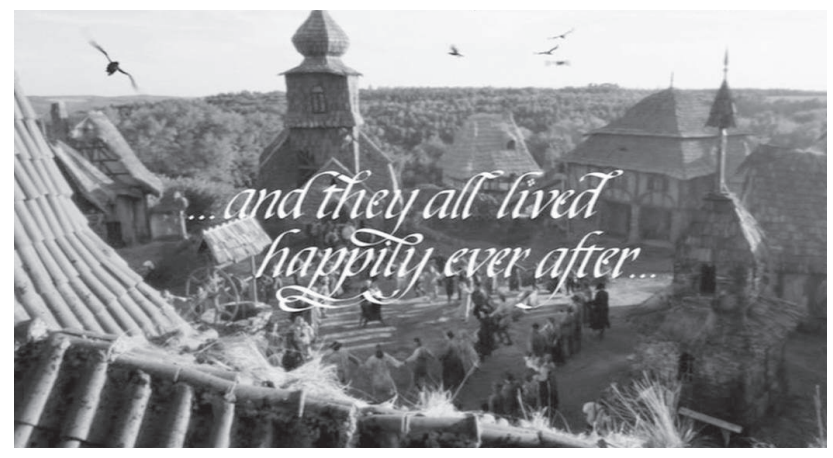

7
23 Vladimir Propp, Morphologie du conte, Paris, Seuil, 1965, p. 112. Son modèle a depuis été remis en question, surtout lorsqu'il est appliqué à des contes qui ne font pas partie de son corpus limité aux seuls textes d'Afanassiev.

24 Ruth Bottigheimer, Fairy Tales. A New History, New York, State University of New York Press, 2009. à nous maintenant. Nous la vivons. Elle est vivante, elle est réelle, elle respire. Et nous pouvons lui donner une fin heureuse». L'œuvre de Jacob et Wilhelm Grimm devient ainsi une partie de l'intrigue d'un film fantastique où l'hésitation provient du fait que la fiction fait irruption dans la réalité.

Au niveau de l'intrigue, le film se distancie également des contes de fées. Vladimir Propp a tenté de définir le genre d'après la structure des récits: "On peut appeler conte merveilleux du point de vue morphologique tout développement partant d'un méfait $(A)$ ou d'un manque $(a)$, et passant par les fonctions intermédiaires pour aboutir au mariage $(W)$ ou à d'autres fonctions utilisées comme dénouement» $\mathbf{2 3}$. Suivant une autre démarche, Ruth Bottigheimer propose une définition assez proche en prenant en considération la "trajectoire narrative» des textes : il existe, selon elle, des contes de fées de rétablissement (restoration fairy tales, à la manière de Cendrillon qui retrouve son rang par le mariage) et des contes de fées d'ascension (rise fairy tales, comme dans le "Maître chat ou le Chat botté» de Perrault où le héros, ne possédant rien d'autre qu'un chat, épouse une princesse) ${ }^{\mathbf{2 4}}$. Aucune des définitions ne s'applique au film de Gilliam, car, bien que pauvres au début, les frères restent pauvres à la fin. L'intrigue correspond plus à certains films fantastiques où un personnage mène une enquête pour comprendre si des événements peuvent s'expliquer de manière rationnelle ou s'ils sont le fait de forces surnaturelles. C'est aussi, par exemple, le cas d'un film écrit par le scénariste de The Brothers Grimm, et sorti lui aussi en 2005, où l'héroïne à premier abord incrédule se confronte à la magie noire: The Skeleton Key. Dans le film de Gilliam, les Grimm sont d'ailleurs engagés (de force) par le général Delatombe pour fournir une explication à des événements apparemment surnaturels et y mettre fin. 


\section{Genèse du livre - importance de l'écrit}

Les films tirés de contes font souvent figurer, parmi les éléments de leur diégèse, les livres desquels l'histoire serait tirée. C'est le cas des trois dessins animés de Disney énumérés ci-dessus, qui mettent en scène un livre (un très bel objet ornementé, semblable aux livres d'étrennes) s'ouvrant au début du film et se refermant à la fin. De plus, ces derniers donnent par moment à lire, en surimpression, du texte faisant le lien entre les parties de l'intrigue et lu simultanément par une voix over. Ce dispositif simule une situation de contage dans laquelle la voix over se substituerait à la voix du narrateur, ou du moins se confondrait avec elle. Il rappelle par là que l'on a affaire à l'adaptation d'un texte écrit.

Le début et la fin de The Brothers Grimm présentent également du texte incrusté dans l'image, reprenant les formules types des contes: «Il était une fois...» ("Once upon a time...»), qui apparaît lettre par lettre pour simuler l'écriture en direct, et «Et ils vécurent heureux pour le restant de leurs jours» ("And they all lived happily ever after») (fig. 6-7). Ce procédé pourrait laisser penser qu'il s'agit également d'un film tiré d'un livre. Le principe est toutefois différent, car il n'y a pas de voix over ni d'autres passages écrits; on ne fait croire à aucun moment que l'on raconte une histoire issue d'un texte écrit. C'est en fait l'histoire du livre lui-même qui est racontée: le film n'est pas né d'un livre, c'est le livre qui est représenté à l'intérieur du film 25. En effet, tout au long de The Brothers Grimm, on peut voir Jake porter un livre et y consigner les histoires et légendes qu'il entend $\mathbf{2 6}$ (fig. 8), ou chercher le titre d'un récit dans la pose d'un artiste cherchant l'inspiration $\mathbf{2 7}$ (fig. 9). Il consulte également son livre en quête de réponses face aux événements qui lui arrivent. L'objet n'est donc pas présenté du côté du lecteur, mais de l'auteur; il ne s'agit pas d'un livre abouti, comme dans les dessins animés de Disney, où l'on pourrait puiser une histoire, mais d'un livre en cours
25 II est d'ailleurs étrange que le menu du dvd allemand (qui diffère du dvd français édité par Metropolitan Filmexport en 2006) présente un livre avec des pages qui se tournent. II ne s'agit en outre pas d'un livre d'étrennes doré comme chez Disney, mais d'un gros livre poussiéreux d'alchimiste qui rappelle plutôt le livre de la reine que l'on trouve à l'intérieur du film, si bien que ce motif tend vers le fantastique. La bandeannonce développe aussi le lien entre le film et le livre en faisant apparaître des mentions écrites sur un fond composé d'un conte des Grimm - le choix du conte, "Les deux frères", exploite là aussi le lien entre fiction et réalité.

26 Dans le village de Marbaden, il écrit par exemple "little red riding cape" (jeu sur le titre anglais du petit chaperon rouge: "Little Red Riding Hood"), ou cet énoncé qui rappelle "Jack et le haricot magique": "Je vais moudre ses os pour faire mon pain" ("l'll grind his bones to make my bread").

$\mathbf{2 7}$ Le titre ("Les Ruines maudites"/ The Cursed Ruins") ne présage pas de bons augures. Comme je le propose plus loin, les Grimm gagneront leur statut d'auteurs après avoir euxmêmes vécu les histoires qu'ils racontent.

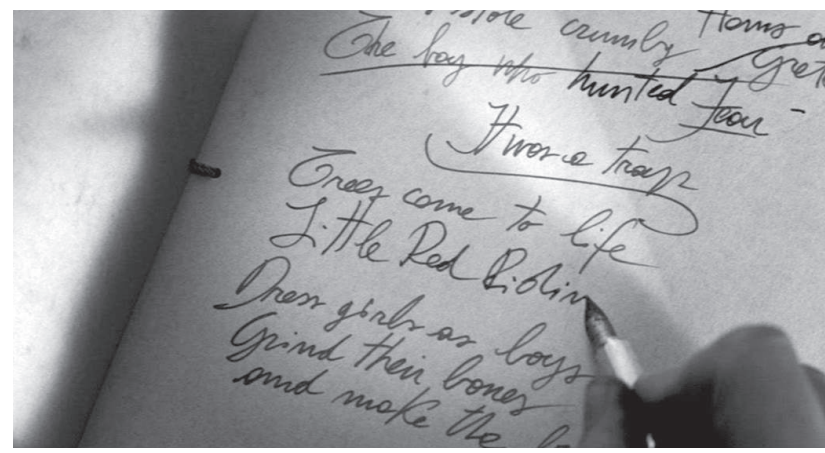

8

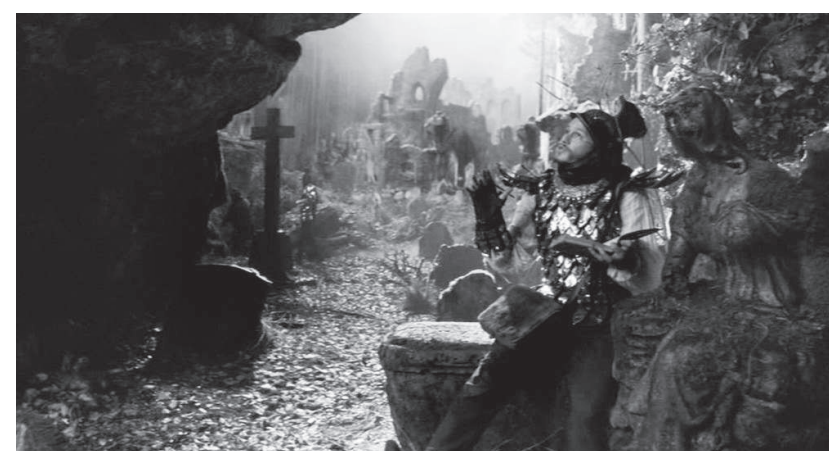

9 
28 Disponible dans les bonus de l'édition dvd Concorde Video. d'établissement qui regroupe des notes éparses. On trouve bien des références à des textes écrits dans le film, mais il ne s'agit que d'allusions : en se promenant dans la forêt, Gretel raconte l'histoire de la maison en pain d'épices telle qu'elle est racontée dans les Contes de l'enfance et du foyer; dans une scène supprimée au montage ${ }^{\mathbf{2 8}}$, un homme lit "Aschenputtel » pendant que Jake murmure les mots en même temps, montrant ainsi qu'il connaît ce texte par cœur.

Le livre est non seulement omniprésent dans le film, mais on suit, en outre, son histoire. Jeté au feu par le général Delatombe s'exclamant: "Et adieu à vos contes. Ils tomberont dans l'oubli.» (fig. 10), il manque d'être brûlé mais échappe aux flammes grâce à Cavaldi, sbire du général d'abord incrédule, puis conquis par les Grimm, qui donne ce conseil aux deux frères : "Et ceci, vous devriez y faire plus attention". Ce livre, sauvé miraculeusement, constitue les futurs Contes de l'enfance et du foyer, dont la première édition paraît en 1812, soit l'année succédant aux événements racontés dans le film si l'on en croit les indications temporelles qui y figurent - une mention écrite prend soin de préciser que la première scène a lieu en 1796, puis que le reste du film se déroule quinze ans plus tard.

Si The Brothers Grimm n'est pas un biopic, il explique toutefois d'où viennent les contes, présentant ainsi en quelque sorte la genèse du livre. Utilisant dans un premier temps le folklore pour duper un peuple trop crédule, les frères peuvent publier le livre après avoir eux-mêmes été au centre de l'histoire. Le film paraît alors comme une initiation, en commençant par une remise en question du surnaturel auquel seul Jake croit, avant que ce dernier, jusqu'alors incompris, ne sauve la situation grâce à ses connaissances et ses croyances, réhabilitant ainsi le surnaturel. Le monde des haricots magiques prend le dessus et les

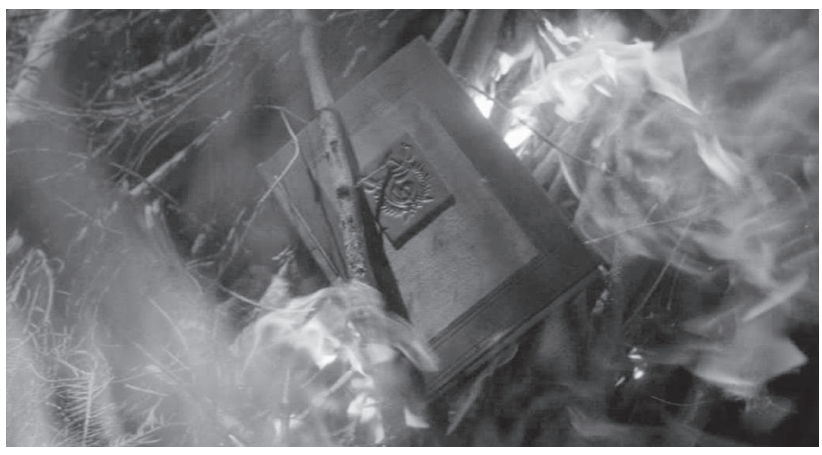


auteurs, ayant vécu ces aventures, deviennent garants de l'authenticité des contes.

Ce scénario permet de faire des protagonistes des sujets agissants, des héros d'un film d'action, qui correspondent plus au genre fantastique que les personnages réels auxquels ils font référence. Le mélange de fiction et de biographie répond également à un phénomène de mode à Hollywood où, comme l'a écrit McCabe, les films "ré-imaginent» la vie de personnages historiques. Le brouillage entre fiction et réalité au cinéma dans une réflexion sur les biopics et les documentaires $\mathbf{2 9}$ coïncide avec le développement de la téléréalité sur le petit écran, qui brouille elle aussi les pistes entre documentaire et fiction. On peut ainsi penser que le genre de biographie fictive mis en œuvre dans The Brothers Grimm est à la fois un refus d'aborder la biographie comme un documentaire et le signe d'une époque qui s'inspire de plus en plus de personnages réels pour développer ses sujets de fiction. Le brouillage des distinctions entre fiction et biographie est enfin typique du postmodernisme, qui se caractérise selon Linda Hutcheon par ce qu'elle appelle la «métafiction historiographique» $\mathbf{3 0}$ et par une dimension autoréflexive. Le miroir de The Brothers Grimm, écran sur lequel se projette une réalité alternative grâce à laquelle la méchante reine manipule les gens - recourant pour ce faire à une technique de marionnettiste -, n'est-il d'ailleurs pas une métaphore du cinéma? $\mathbf{3 1}$ Ce monde de faux-semblants, que l'on retrouve au début du film dans la mise en scène d'effets visuels dont usent les personnages des frères Grimm pour duper les villageois, fait écho au travail de Gilliam lui-même œuvrant à présenter le surnaturel de manière réaliste, et à transformer des philologues allemands en héros de film d'action fantastique.
29 Voir par exemple I'm not there (Todd Haynes, 2006). Dans le monde littéraire, le même type de réflexion a lieu autour des concepts d'autobiographie et d'autofiction.

30 Linda Hutcheon, A Poetics of Postmodernism: History, Theory, Fiction, New York/London, Routledge, 1988, p.ix. L'auteure considère justement Brazil comme typiquement postmoderne dans sa "re-conception ironique de l'histoire" qui se manifeste par les références parodiques à d'autres films (pp. 4-5).

31 La question du miroir est au centre de l'article de Susan Cahill, "Through the Looking Glass: Fairy-Tale Cinema and the Spectacle of Femininity in Stardust and The Brothers Grimm ", in Marvels \& Tales, Detroit, 2010, 24: 1, pp. 57-67. 